\title{
Condiciones oceanográficas durante la fase de relajación de un evento de surgencia invernal frente a Punta Lengua de Vaca, Coquimbo
}

\author{
Julio Moraga, Eugenia Valdebenito y José Rutllant* \\ Departamento de Biología Marina, Facultad de Ciencias del Mar, \\ Universidad Católica del Norte, Casilla 117. Coquimbo-Chile \\ E-mail: jmoraga@ucn.cl. \\ *Departamento de Geofísica, Facultad de Ciencias Físicas y Matemáticas \\ Universidad de Chile.
}

Recibido: 26 julio 1999; versión corregida: 27 diciembre 2000; aceptado: 20 marzo 2001

\begin{abstract}
RESUMEN. Entre el 10 y 17 de agosto de 1990 se efectuaron estaciones oceanográficas en el área frente al foco de surgencia de Punta Lengua de Vaca mediante CTD y botellas hasta $350 \mathrm{~m}$ de profundidad y una distancia de 12 millas náuticas de la costa (aproximadamente $22 \mathrm{~km}$ ). Se analizan e interpretan, mediante perfiles T-S y estimaciones geostróficas, las características y dinámica de las aguas costeras y oceánicas en este período. Las observaciones comprendieron un período de relajación y posterior intensificación de los vientos favorables a la surgencia observados in situ mediante una estación meteorológica automática.

La diferencia en el comportamiento de los campos de temperatura y salinidad en las estaciones próximas a la costa respecto de las oceánicas se explica por la intrusión de masas de agua y la influencia de ondas internas de gravedad. Estimaciones geostróficas referidas a $300 \mathrm{db}$ indican corrientes del orden de $10 \mathrm{~cm} * \mathrm{~s}^{-1}$ hacia el norte en los primeros $100 \mathrm{~m}$ y hacia el sur bajo esa profundidad, consistentes con un esquema clásico de surgencia costera. Se analiza además la influencia de la surgencia generada frente a Punta Lengua de Vaca en las bahías de Tongoy y Guanaquero, ubicadas al norte de ese foco.
\end{abstract}

Palabras claves: surgencia costera, intrusión de masas de agua, ondas internas, relajación de vientos.

\section{Oceanographic conditions during a relaxation phase of a winter upwelling event off Point Lengua de Vaca, Coquimbo}

\begin{abstract}
Oceanographic stations in a $12 \mathrm{~nm}$ transect off the upwelling focus of Punta Lengua de Vaca and adyacent area were occupied using CTD and bottles to $350 \mathrm{~m}$ depth on August 10 to 17, 1990. The characteristics and dynamics of coastal and oceanic waters are analyzed and interpreted by means of T-S profiles and geostrophic speed estimates. The observational period coincided with a relaxation of the coastal wind followed by an upwelling-favorable wind event, as observed "in situ" by an automatic metereological station.

Differences of the temperature and salinity behavior at the coastal stations with respect to the oceanic ones are explained by intrusion of oceanic water masses and the influence of internal gravity waves. Geostrophic estimates relative to the $300 \mathrm{db}$ level indicate northward currents of the order of $10 \mathrm{~cm} * \mathrm{~s}^{-1}$ in the first $100 \mathrm{~m}$ depth layer and southward ones under that level, consistent with the classical coastal upwelling scheme. The influence of the upwelling focus on the Tongoy and Guanaquero bays located north of Punta Lengua de Vaca is analyzed.
\end{abstract}

Key words: coastal upwelling, water masses intrusion, internal gravity waves, wind relaxation.

\section{INTRODUCCIÓN}

Las condiciones oceanográficas de la zona costera de Coquimbo se han caracterizado por su alta variabilidad espacio-temporal, la que ha sido atribuida al efecto de la batimetría y vientos que la afectan (Moraga et al., 1994). En la zona comprendida entre los 29 y $31^{\circ} \mathrm{S}$ se han definido dos focos de surgencia, uno al sur frente a $\operatorname{los} 30,5^{\circ} \mathrm{S}$, al sur de Punta Len- gua de Vaca (Neshyba y Méndez, 1976; Fonseca y Farías, 1987) y otro al norte, frente a los $29^{\circ} \mathrm{S}$ (Acuña et al., 1989; Moraga, 1996).

Punta Lengua de Vaca corresponde a una península de 2,4 km de longitud orientada al norte, cerrando por el oeste la bahía Tongoy. Según Acuña et al., (1989), Moraga y Olivares (1993) y Moraga et 
al., (1994), esta punta sería la parte sur de una gran bahía que contiene un sistema de bahías más pequeñas (Tongoy, Guanaquero, La Herradura de Guayacán y Coquimbo), limitado al norte por Punta Pájaros, islas Choros y Damas, y al este por la isóbata de $1000 \mathrm{~m}$. En ambos extremos aparecen focos de surgencia apreciados en las imágenes de satélite (Fonseca y Farías, 1987; Acuña et al., 1989).

En el marco de los programas de estudio de la zona costera entre la Universidad Católica del Norte, Facultad de Ciencias del Mar y el Comité de las Ciencias del Mar de la Universidad de Chile, se realizó una campaña en agosto de 1990, para estudiar la zona de Punta Lengua de Vaca. El objetivo era realizar una investigación multidisciplinaria en un área de surgencia que considerara las variables físicas y químicas, componentes planctónicas y forzantes meteorológicos locales. Valdebenito (1997) analizó la influencia de las condiciones físicas y químicas en el comportamiento del zooplancton frente a Punta Lengua de Vaca en esa campaña de 1990.

Este trabajo pretende, en primer lugar, describir las características hidrográficas observadas durante el estudio efectuado entre el 10 y 17 de agosto, y realizar un análisis de la dinámica oceanográfica que presenta el área desde la costa hasta $22 \mathrm{~km}$ mar afuera.

\section{MATERIALES Y MÉTODOS}

El estudio se realizó frente a Punta Lengua de Vaca (PLV en adelante) $\left(30^{\circ} 16^{\prime} \mathrm{S}\right)$ y frente a las bahías Tongoy y Guanaquero, a bordo de L/I Stella Maris II. Los días 10 al 12 de agosto de 1990 se efectuaron cortes oceanográficos este-oeste frente a PLV con estaciones a $2,4,6,8,10$ y $12 \mathrm{mn}$ de la costa (aproximadamente a 3,7; 7,4; 11,1; 14,8; 18,5 y 22,2 $\mathrm{km}$ la costa) (Fig. 1 Corte A). El 14 de agosto se realizó un corte frente a Guanaquero hasta $22 \mathrm{~km}$ de la costa (Fig.1 Corte B); el 15 de agosto el corte se ubicó frente a la boca de bahía Tongoy y las muestras se tomaron con botellas, sólo la estación a 8 millas alcanzó una profundidad hasta los $300 \mathrm{~m}$ (Fig.1 Corte C). El 16 de agosto la orientación del corte siguió la línea de la boca de la bahía desde PLV, y el 17 de agosto se realizó un corte partiendo del centro de la bahía Tongoy hacia el oeste, limitando la profundidad en los $120 \mathrm{~m}$ (Fig.1. Corte D).

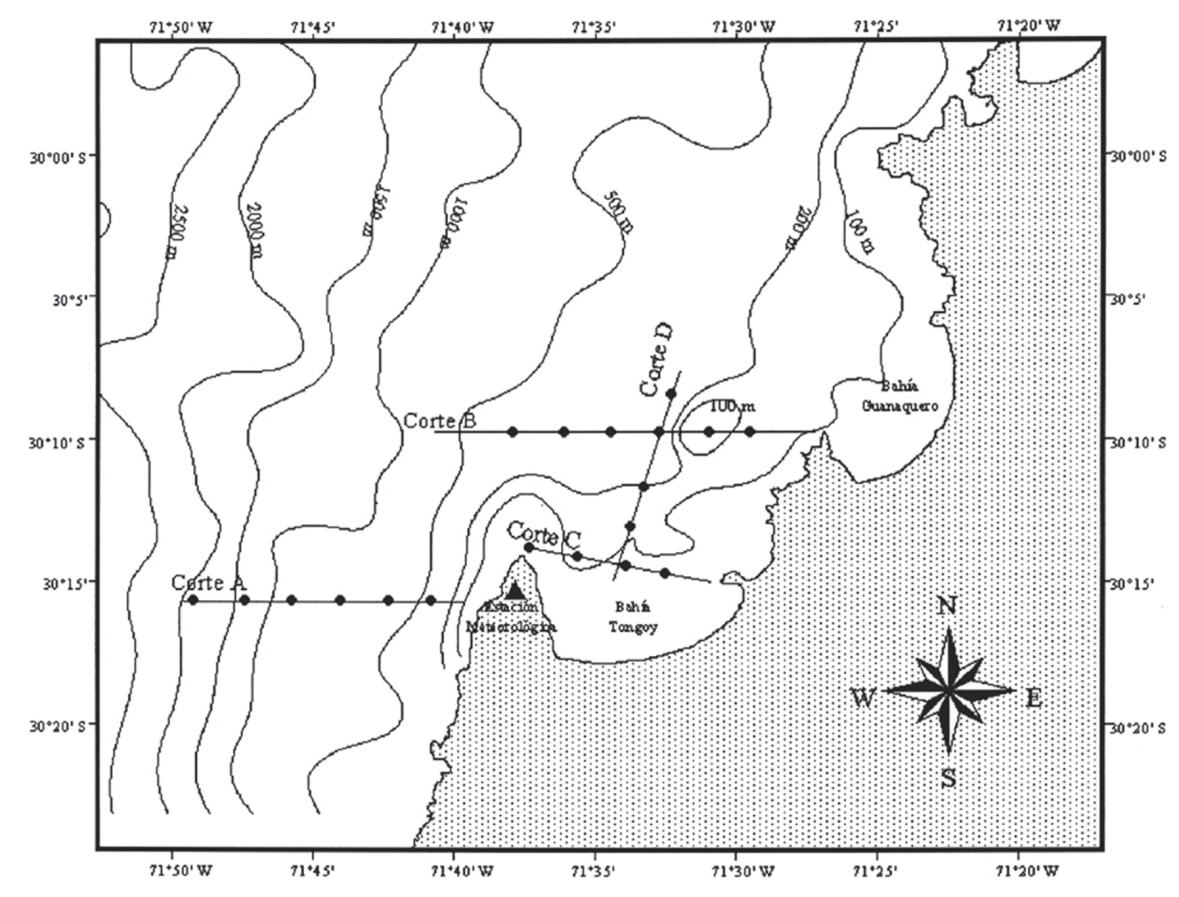

Figura 1. Área de estudio. Se indican las isóbatas de $100 \mathrm{~m}, 200 \mathrm{~m}$ y $1000 \mathrm{~m}$ próximas al borde costero y la ubicación de los cortes oceanográficos. La estación meteorológica está localizada frente al Corte A.

Figure 1. Study area. The $100 \mathrm{~m}, 200 \mathrm{~m}$ and $1000 \mathrm{~m}$ isobaths and the oceanographic sections are drawn. The meteorological station is located in front of section $A$. 
En cada estación frente a PLV, se llevó a cabo un lance de CTD hasta los $350 \mathrm{~m}$ como máxima profundidad, y lances con botellas Niskin y Nansen en algunas de las estaciones (2, 6, 8 y $12 \mathrm{mn}$ ). El 14 de agosto se realizaron sólo lances con CTD y los días siguientes se efectuaron lances con botellas, debido a problemas de funcionamiento del CTD. Los datos del CTD se corrigieron y suavizaron. Los cálculos de densidad potencial y geostróficos se efectuaron de acuerdo a las ecuaciones de UNESCO (1981).

Para la aplicación de la aproximación geostrófica, se usó como nivel de referencia $300 \mathrm{~m}$ de profundidad, extrapolando en las estaciones de menor profundidad la topografía dinámica de las estaciones más próximas. Niveles de referencia del mismo orden han sido empleados frente a California por Huyer y Kosro (1987) entre otros. Los cálculos geostróficos a partir de los datos de CTD se realizaron para las estaciones entre 4 y $8 \mathrm{mn}$, y entre 8 y 12 $\mathrm{mn}$, considerando que la variación de la distancia entre estaciones generaría un error mayor si se usaran estaciones contiguas, tanto por el sistema de posicionamiento utilizado como por la influencia de la deriva durante la realización del lance. La posición de cada estación fue determinada antes de cada lance de CTD con un radar Furuno y el error estimado es del orden de $8 \%$ en el cálculo de la velocidad geostrófica.

Para separar los factores advectivos de los efectos de las ondas internas y la influencia de estructuras asociadas a frentes, se realizó un análisis isentrópico planteado por Fedorov (1978) y utilizado por Fisher et al. (1989), en el cual la variabilidad termosalina queda limitada a la intrusión de masas de agua. El análisis isentrópico elimina el efecto de las ondas internas en general, al considerar la variación temporal y espacial de las isotermas e isohalinas en función de la densidad potencial.

Los datos de viento y presión atmosférica fueron obtenidos de una estación meteorológica autónoma Campbell, con lecturas cada $15 \mathrm{~s}$ y registro de valores promedios cada $30 \mathrm{~min}$. La estación fue ubicada aproximadamente a $15 \mathrm{~m}$ sobre el nivel del mar en PLV, en la latitud indicada, frente al corte oceanográfico. Con el objetivo de ampliar la representatividad del evento de surgencia analizado, se consideraron los registros diarios de viento para el período 1990-1998 a través del índice diario de surgencia definido como seudoesfuerzo de la componente meridional (positivo de sur a norte) del viento norte-sur entre las 12 y $24 \mathrm{hr}$ de cada día.

\section{RESULTADOS}

\section{Forzamiento local de la surgencia}

El registro de vientos durante agosto de 1990 (Fig. 2 ), incluye eventos intensos de vientos favorables a la surgencia (vientos al norte, con velocidades que exceden los $\left.8 \mathrm{~m} * \mathrm{~s}^{-1}\right)$. Sin embargo, durante los días 10 y 12 (Fig. 2a) la intensidad de la componente $\mathrm{v}$ (meridional) sólo alcanza los $4 \mathrm{~m} * \mathrm{~s}^{-1}$, correspondiendo a un período de relajación de la surgencia. En la figura 2a, se observa también la variación temporal de la presión atmosférica a nivel del mar; una mínima presión (baja costera) culmina el 8 de agosto, subiendo $9 \mathrm{hPa}$ en $36 \mathrm{hr}$, y alcanzando un valor de $1.020 \mathrm{hPa}$ 5,5 días después del mínimo anterior. Estos cambios de presión se asocian con los cambios de vientos, estando los días más intensos de viento norte asociados con el descenso de la presión luego de ocurrida la presión máxima, con un desfase del orden de un día. El día 15 se observa una nueva intensificación de los vientos del sur, con una duración menor al pulso anterior.

Un análisis espectral de todo el registro hasta 1998 (Fig. 2b), muestra que el período entre 7 y 8 días $(170 \mathrm{hr})$ es el más significativo dentro del espectro, lo que permite considerar que el tiempo de estudio de agosto se enmarcó dentro de un período típico de reforzamiento-relajación de los vientos.

\section{Condiciones oceanográficas frente a Punta Lengua de Vaca}

Las figuras 3 y 4 muestran los cortes transversales de temperatura, salinidad frente a PLV del 10 al 12 de agosto. El primer día se observa entre los 11 y 20 $\mathrm{km}$, a profundidades entre 60 y $120 \mathrm{~m}$ un cambio térmico (Fig. 3a), junto a un gradiente salino horizontal bien definido de 0,16 psu entre los 10 y 15 km (Fig. 4a). Se distingue un cuerpo de agua de salinidad 34,72 psu y temperatura entre 10,8 y $11,8^{\circ} \mathrm{C}$, entre 60 y $200 \mathrm{~m}$ de profundidad (masa de agua $\mathrm{A}$ ), que se extiende desde la costa hasta los 18 $\mathrm{km}$. Por otra parte, una masa de agua de salinidad menor de 34,56 psu y temperatura de $11,2^{\circ} \mathrm{C}$ está centrada entre los 15 y $20 \mathrm{~km}$, entre 40 y $100 \mathrm{~m}$ de profundidad (masa de agua B). Estas dos masas de agua, A y B producen el cambio térmico y salino descrito anteriormente. Bajo los $240 \mathrm{~m}$, se hunden hacia la costa las isolíneas tanto de temperatura como salinidad y se observa una capa de temperatura uniforme "termo-stad" de 11,6 a $11,8^{\circ} \mathrm{C}$ entre los 5 y $12 \mathrm{~km}$, de $40 \mathrm{~m}$ de espesor. 
a)
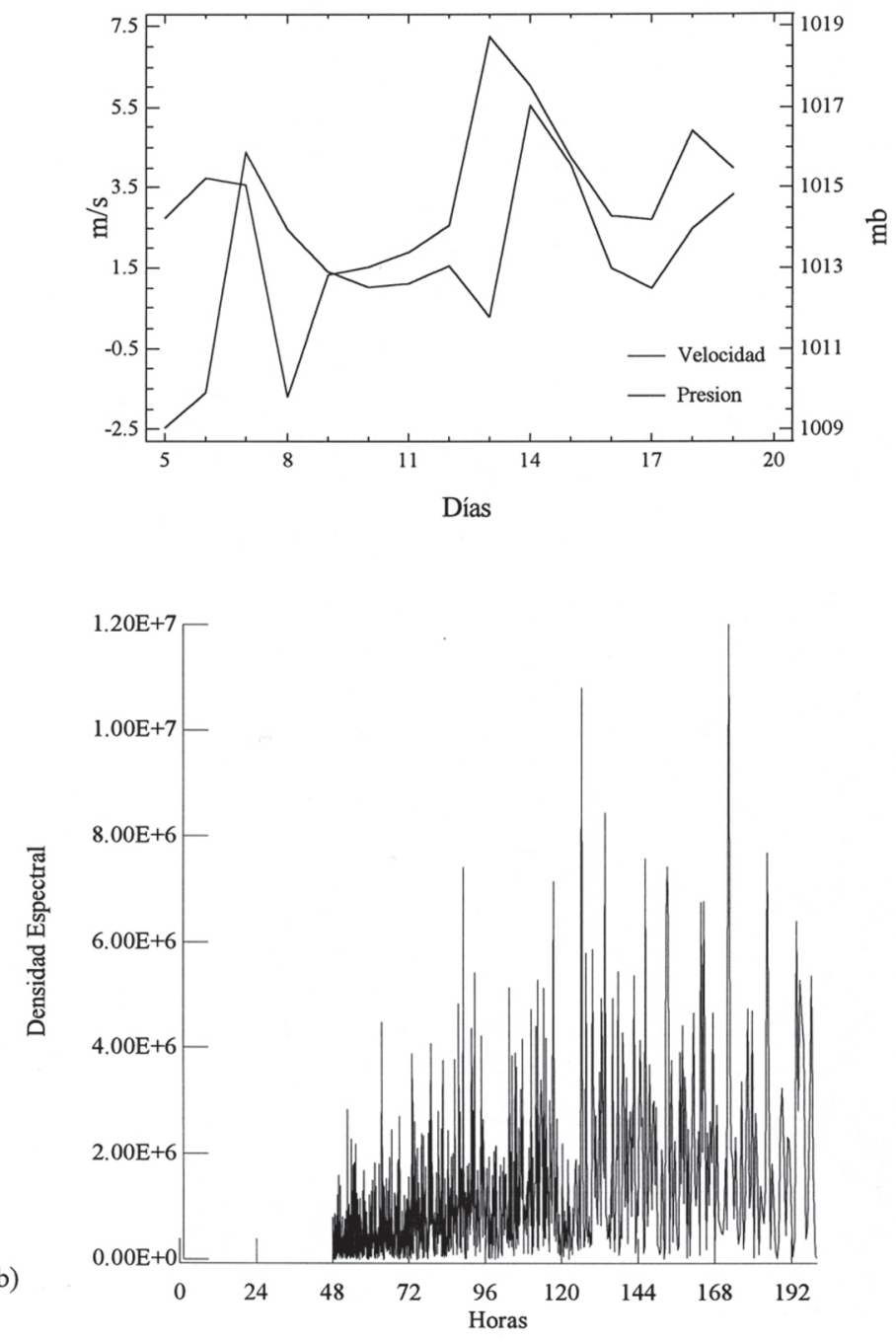

Figura 2. a) Componente norte-sur de vientos registrados en la estación meteorológica en Punta Lengua de Vaca en agosto de 1990 (línea segmentada) y presión atmosférica suavizada a nivel del mar durante los mismos días (línea continua). b) Espectro del seudoesfuerzo de la componente norte-sur del viento entre los años 1990 y 1998 (escala horizontal cph).

Figure 2. a) Meridional component of wind recorded at the meteorological station of Punta Lengua de Vaca on August 1990 (segmented line), and smoothed sea-level atmospheric pressure during the same period (continuous line). b) Spectrum of the meridional component of the daily pseudostress of the wind between 1990 and 1998 (horizontal scale cph).
El día 11 el cuerpo de agua de 34,72 psu aumenta de espesor ( 80 a $260 \mathrm{~m}$ ) extendiéndose hasta los $20 \mathrm{~km}$, no observándose la masa de agua B (Fig. 4b). Entre los 40 y $100 \mathrm{~m}$ se hunde una haloclina desde los $5 \mathrm{~km}$ hacia los $22 \mathrm{~km}$. Una suave termoclina superficial se presenta en los niveles superiores de la columna de agua (40 $\mathrm{m}$ de profundidad, Fig. 3b) distinguiéndose el hundimiento de isolíneas hacia la costa bajo los $240 \mathrm{~m}$, como el día anterior.

El día 12 vuelve a aparecer la masa de agua B entre 17 y $23 \mathrm{~km}$ desde la costa, con temperatura de $11,4^{\circ} \mathrm{C}$ y salinidad de 34,46 psu entre 60 y 100 m, y el agua más salina (masa A) disminuye su ancho y espesor, apareciendo un gradiente termohalino en todo el corte (Figs. 3c y 4c). Este gradiente ubicado entre los $80 \mathrm{y}$ $90 \mathrm{~m}$ en los $22 \mathrm{~km}$ sube hasta los 20-40 m a $5 \mathrm{~km}$ de la costa. Se distingue la capa homotermal de un espesor menor a $60 \mathrm{~m}$ centrada en los $5 \mathrm{~km}$, de $6 \mathrm{~km}$ de ancho. El gradiente termohalino muestra la intrusión de agua de baja salinidad hacia la costa en los $100 \mathrm{~m}$ desde 22 km ("interleaving") hasta $10 \mathrm{~km}$ desde la costa. Bajo los $200 \mathrm{~m}$, las isolíneas de temperatura y salinidad se hunden hacia la costa y se observa una inversión térmica y salina a $18 \mathrm{~km}$ de la costa y $80 \mathrm{~m}$ de profundidad.

El campo de masa (Figs. 5a, 5 b y 5 c) muestra que la isopicna de 26,5 (unidades de densidad potencial sigma theta) es la más horizontal, y una suave picnoclina superficial limitada por la isopicna de 26,4. Se observa que la isopicna de 26,5 varía unos 20 $\mathrm{m}$ en profundidad entre el $10 \mathrm{y}$ 12 de agosto, con un cambio de curvatura. Esta variación temporal indicaría una oscilación de la 

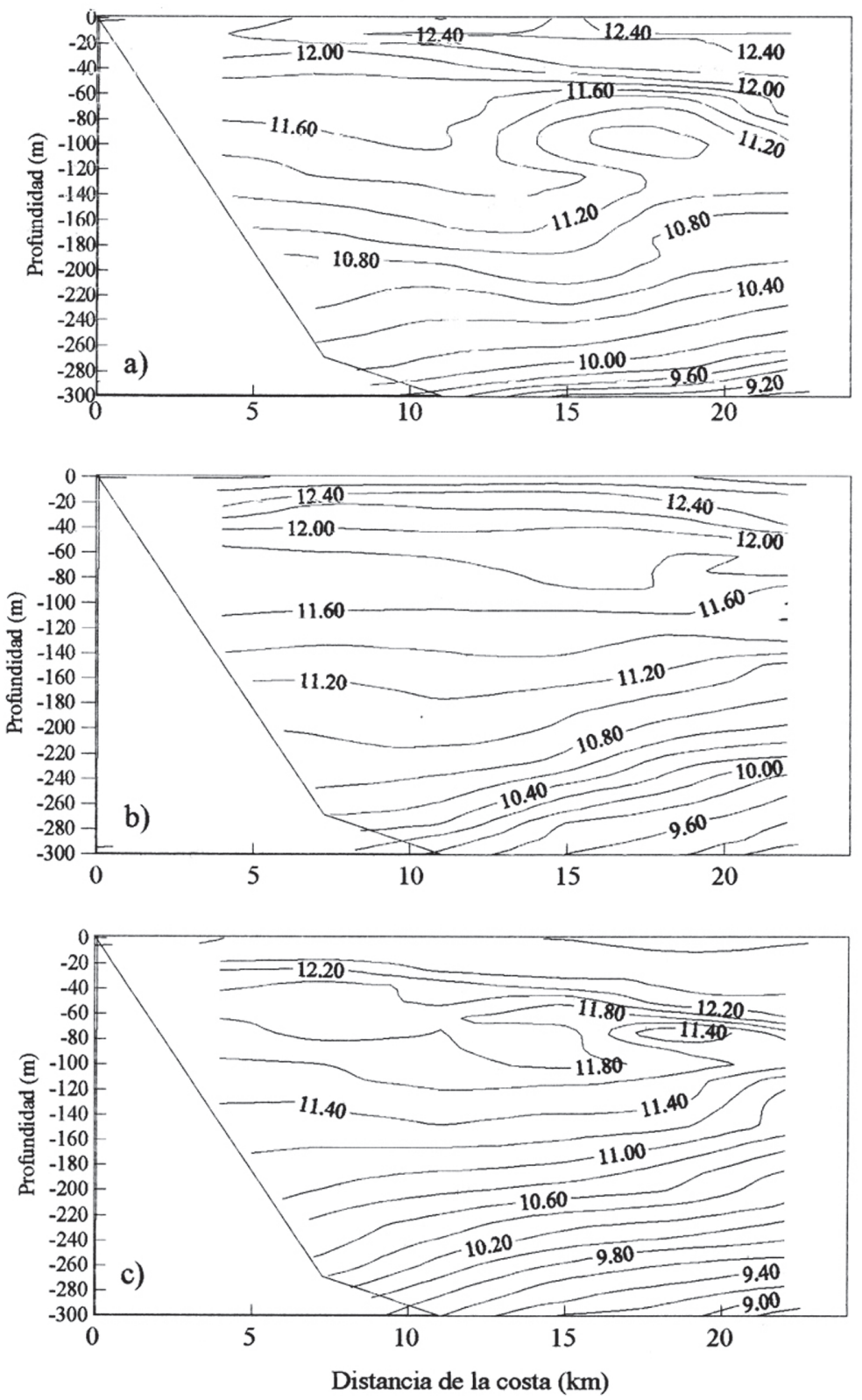

Figura 3. Distribución de isotermas $\left({ }^{\circ} \mathrm{C}\right)$ para el corte $A$ efectuado frente a Punta Lengua de Vaca: a) 10 de agosto, b) 11 de agosto, c) 12 de agosto de 1990. Escala horizontal en $\mathrm{km}$.

Figure 3. Isotherm $\left({ }^{\circ} \mathrm{C}\right)$ distribution of section $A$ in front of Punta Lengua de Vaca: a) August 10, b)August 11 and c) August 12, 1990. Horizontal scale in $\mathrm{km}$. misma por el paso de una onda interna. Las isopicnas menores a la señalada, en general, se levantan hacia el borde continental y las mayores se hunden. Se observa, por consiguiente un gradiente horizontal de densidad con diferente signo sobre la isopicna de 26,5 y bajo la misma.

En las curvas T-S (Fig. 6) se distinguen cambios temporales en los niveles superiores de la columna de agua. Estos cambios se interpretan como intrusión de masas de agua (masas A y B descritas anteriormente) y el efecto del paso de ondas internas de gravedad que modifican las curvas T-S. El gráfico de las estaciones cercanas a la costa (estaciones a 2 y $4 \mathrm{mn}$ ) muestra una variación casi nula (Fig. 6a); las estaciones a 6 y $8 \mathrm{mn}$ un cambio mínimo (Fig. 6b), y las estaciones restantes muestran gran variabilidad hasta los $150 \mathrm{~m}$ de profundidad aproximadamente (Fig. 6c).

\section{Condiciones oceanográficas al norte de Punta Lengua de Vaca}

Las condiciones hidrográficas del día 14 frente a Guanaquero (Fig. 6), tienen un comportamiento similar al observado frente a PLV los días anteriores. El núcleo de agua más salina ( $>34,76$ psu masa de agua $\mathrm{A}$ ) más cercano a la costa con temperaturas entre 10,0 y $11,6^{\circ} \mathrm{C}$ se ubica entre los 60 y $260 \mathrm{~m}$ en su máximo espesor, y hasta los 20 km (Figs. 7a y 7b). Una haloclina de 0,16 psu de rango de variación sobre la masa de agua anterior se levanta hacia la costa, dejando un núcleo menos salino en el sector oceánico de 34,52 psu (masa de agua B), asociado a inversiones térmicas. La isopicna de 26,5 se levanta hacia la costa, las isopicnas mayores a 26,6 se hun- 

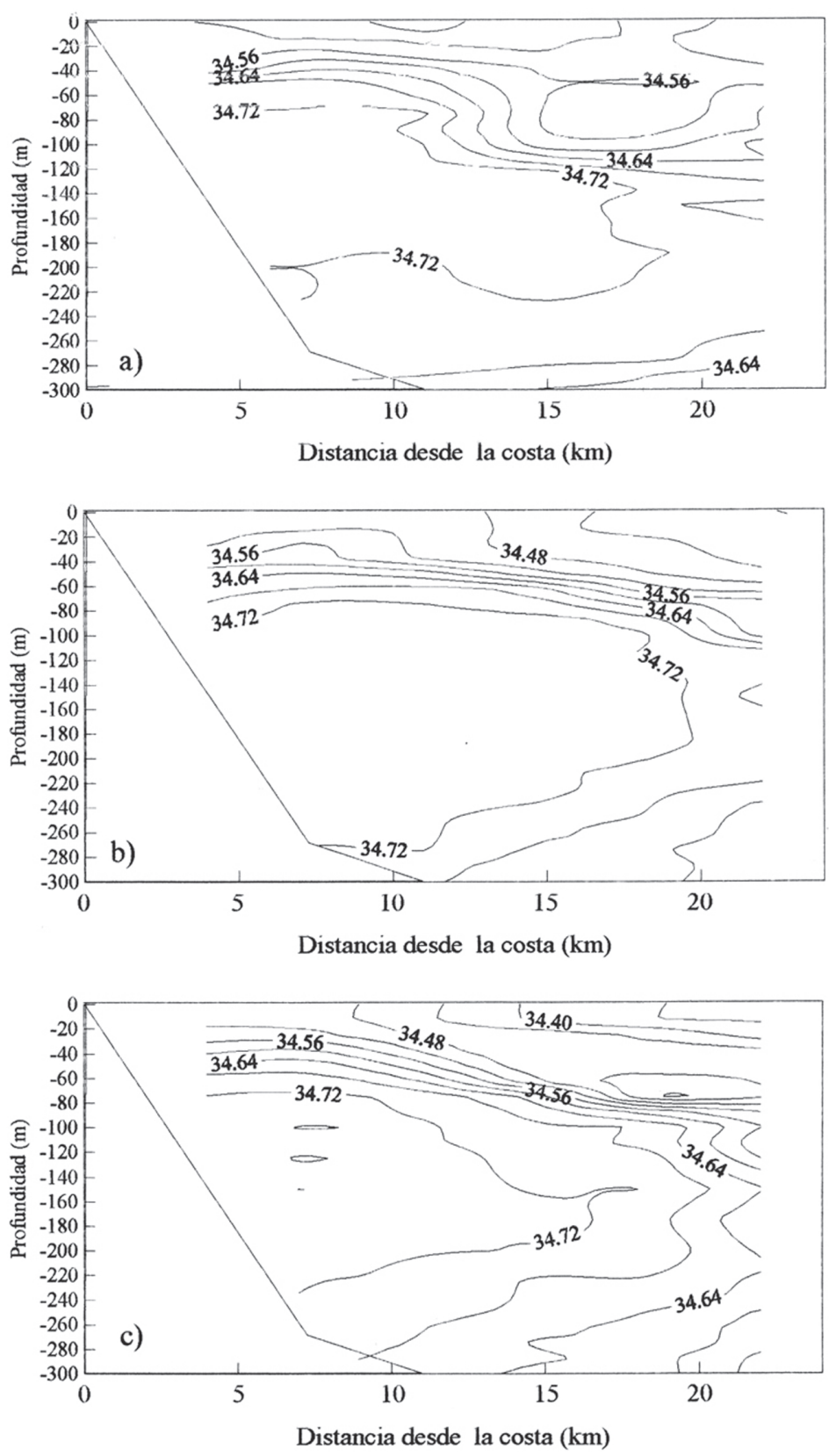

Figura 4. Distribución de isohalinas (psu) para el corte A efectuado frente a Punta Lengua de Vaca: a) 10 de agosto, b) 11 de agosto, c) 12 de agosto de 1990. Escala horizontal en $\mathrm{km}$.

Figure 4. Isohaline distribution (psu) distribution of section A in front of Punta Lengua de Vaca: a) August 10, b)August 11 and c) August 12, 1990. Horizontal scale in $\mathbf{k m}$. den hacia la costa y las menores a 26,5 siguen la tendencia de elevarse hacia el continente.

Una característica observada al norte de PLV desde el día 15 en adelante (Cortes C y D), es la presencia de la isopicna de 26,5 solamente en las estaciones fuera de bahía Tongoy. El hundimiento hacia la costa de la isopicna del día 16, contrasta con la elevación del día 14 (Fig. 7c)

El análisis isentrópico indica una variación temporal de las isotermas de 12,0, 11,5 y 11,0 ${ }^{\circ} \mathrm{C}$, y muestra la intrusión de aguas de diferente condición térmica desde el sector oceánico. Por otra parte, las aguas menos salinas se desplazan a nivel superficial los días 11 y 12 (34,5 psu), acercándose hacia la costa, desde los $13 \mathrm{~km}$ hasta $8 \mathrm{~km}$. A nivel subsuperficial, entre la isopicnas de 26,40 y 26,70, el núcleo de mayor salinidad $(34,74 \mathrm{psu})$, pegado a la costa desaparece el día 12, dejando aguas de 34,72 psu en esa profundidad. Esto significa que la entrada de agua de condiciones térmicas diferentes se presenta entre 40 y $140 \mathrm{~m}$ de profundidad (Fig. 4), de aguas menos salinas entre la superficie y 60 $\mathrm{m}$, y aguas más salinas entre 80 y $260 \mathrm{~m}$ de profundidad.

La presencia de ondas internas de gravedad durante el experimento se observa en las variaciones de profundidad del orden de $20 \mathrm{~m}$ de la isopicna de 26,5. Estas ondas afectan el campo de masa y son causantes de parte de la variación observada en las curvas T-S de las estaciones más alejadas de la costa.

\section{Flujos geostróficos}

El 10 de agosto en la zona entre 4 y 8 mn de la costa (Fig. 8a), se 

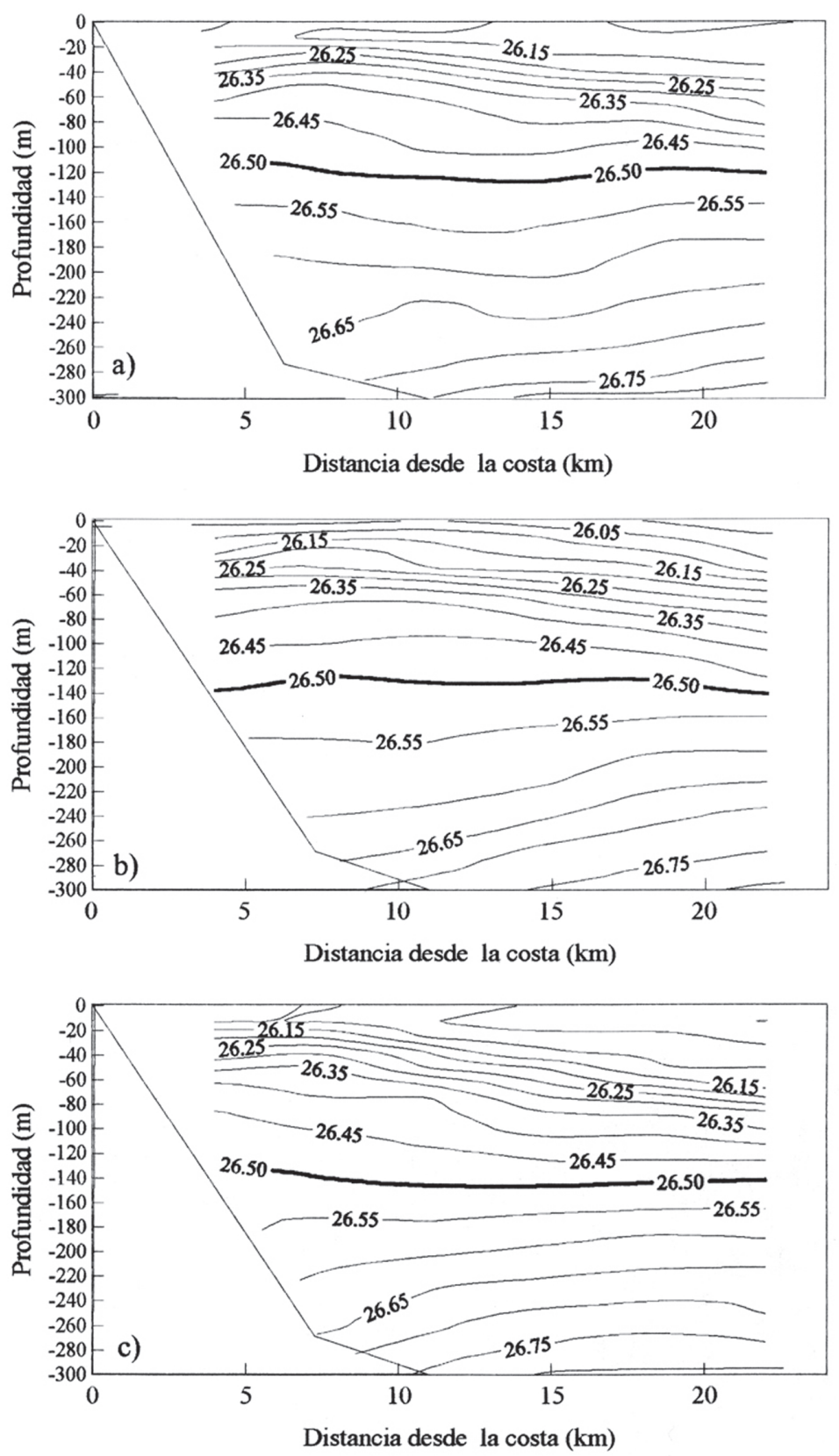

Figura 5. Distribución de isopicnas (densidad potencial), para el corte A efectuado frente a Punta Lengua de Vaca: a) 10 de agosto, b) 11 de agosto, c) 12 de agosto de 1990. Escala horizontal en $\mathbf{~ k m}$.

Figure 5. Isopycnal distribution (potential density), distribution of section A in front of Punta Lengua de Vaca: a) August 10, b)August 11 and c) August 12, 1990. Horizontal scale in km. observa un flujo superficial al norte hasta los $140 \mathrm{~m}$ aproximadamente, bajo esta profundidad el flujo es muy débil ( $<0,1 \mathrm{~m} * \mathrm{~s}$ $\left.{ }^{1}\right)$ y tiende al sur centrándose a $220 \mathrm{~m}$ de profundidad. En la estación entre 8 y $12 \mathrm{mn}$ de la costa (Fig. 8b) no se observa el flujo superficial al norte, encontrándose un flujo al sur centrado a $100 \mathrm{~m}$ de profundidad con velocidades de aproximadamente $0,1 \mathrm{~m} * \mathrm{~s}^{-1}$.

El 11 de agosto entre 4 y 8 mn de la costa (Fig. 8c) no se observa el flujo superficial al norte, presentándose sólo un flujo al sur entre los 60 y $220 \mathrm{~m}$ de profundidad con velocidades de $0,1 \mathrm{~m} * \mathrm{~s}^{-1}$. Entre 8 y $12 \mathrm{mn}$ de la costa, se detecta un flujo superficial al norte ubicado sobre los $40 \mathrm{~m}$ de profundidad, bajo la cual el agua se dirige al sur con velocidades $>0,1 \mathrm{~m} * \mathrm{~s}^{-1}$ (Fig. 8d).

El 12 de agosto reaparece el flujo superficial al norte (entre 4 y $8 \mathrm{mn}$ ) y bajo los $60 \mathrm{~m}$ el flujo al sur de $0,1 \mathrm{~m} * \mathrm{~s}^{-1}$, centrando su mayor velocidad entre $150 \mathrm{y}$ $200 \mathrm{~m}$ de profundidad (Fig. 8e). En las estaciones más alejadas, se observa el flujo superficial al norte que alcanza hasta los $80 \mathrm{~m}$, bajo el cual aparece un débil flujo al sur centrado en los $100 \mathrm{~m}$ de profundidad (Fig. 8f).

La evolución oceanográfica del evento de surgencia aparece evidente en los cambios salinos más que en los cambios térmicos propios de un período invernal en que la columna de agua se enfría.

\section{DISCUSIÓN}

Las condiciones térmicas y salinas observadas en este estudio, son propias de una zona afecta- 

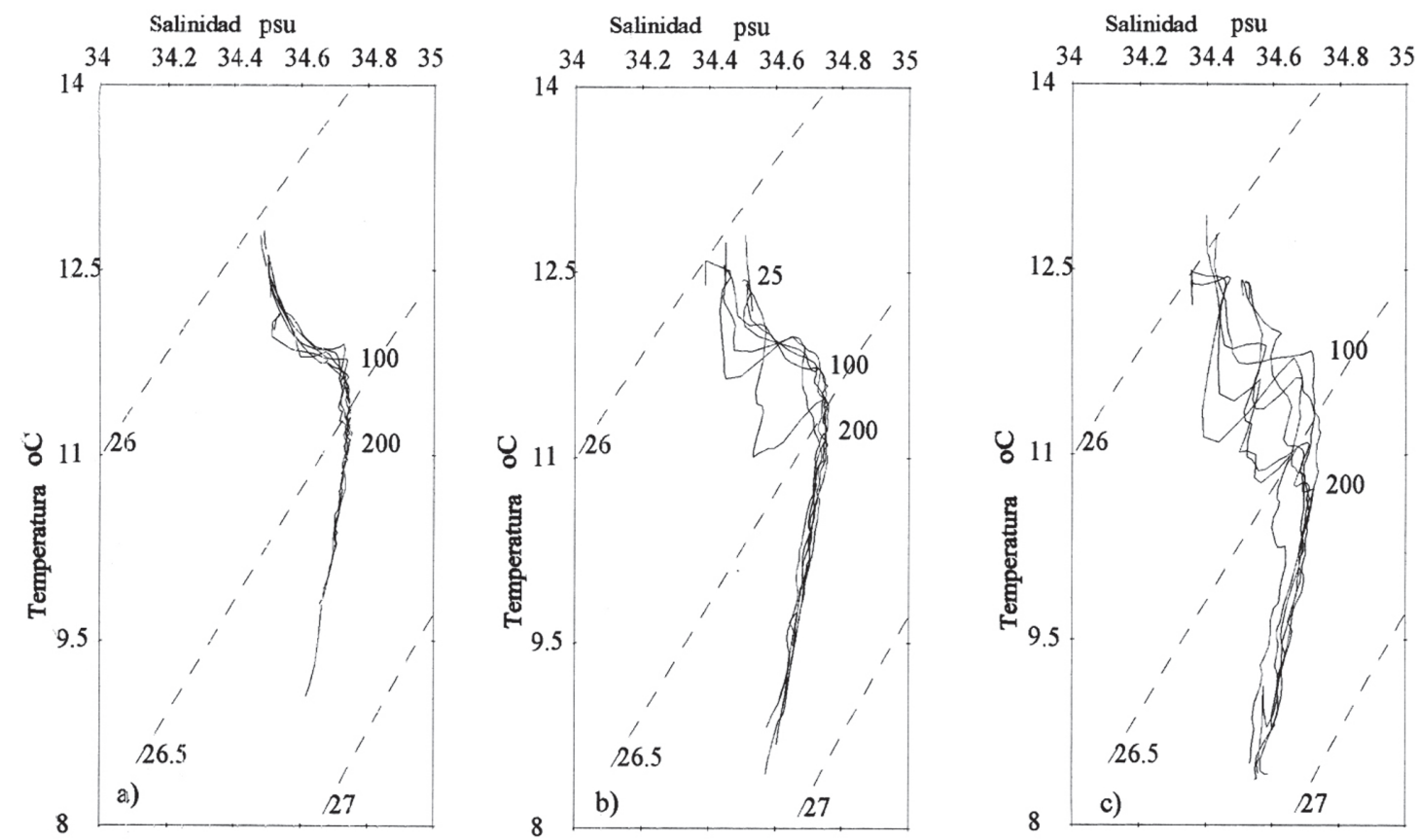

Figura 6. Curvas T-S para las estaciones: a) 2 y 4 millas, b) 6 y 8 millas, y c) 10 y 12 millas para los días 10-12 frente a Punta Lengua de Vaca.

Figure 6. T-S plots for the stations a) 2 and $4 \mathrm{~nm}$, b) 6 and $8 \mathrm{~nm}$, and c) 10 and $12 \mathrm{~nm}$ for August 10 to 12 in front of Punta Lengua de Vaca.

da por surgencia costera en latitudes medias (Johnson et al., 1980). La falta de otros cortes en forma radial, impide estudiar el efecto de la punta sobre la surgencia y por lo tanto considerar la vorticidad relativa como causa de la intensificación del proceso de surgencia. Sin embargo, el efecto del fondo sobre la circulación de las aguas se puede estimar a través de $\mathrm{f} / \mathrm{h}$ (con $\mathrm{f}=$ parámetro de Coriolis, $\mathrm{y} \mathrm{h}=$ profundidad del lugar) (Mellor, 1996), en la zona costera frente a PLV, el valor estimado es muy pequeño debido a que no existe plataforma continental (Fig. 1).

La presencia de un núcleo de mayor salinidad centrado cerca del borde costero (masa de agua A), corresponde en sus características a la masa de agua ecuatorial subsuperficial que es transportada hacia el sur y cuya velocidad debería intensificarse por efecto de la surgencia activa, debido al proceso de ajuste geostrófico generado por el transporte superficial hacia el oeste de Ekman.

Los cambios observados en los campos termohalinos se explican considerando la intrusión de masas de agua y la influencia de ondas internas con un período de días. La posible asociación de la surgencia costera con el paso de ondas atrapadas a la costa no se ha incluido, pues se carece de mediciones directas de corrientes y de registro de nivel del mar (Pizarro et al., 1994). Moraga et al. (1994) eliminan la influencia de la ondas internas en un estudio de la dinámica frente a los $30^{\circ} \mathrm{S}$ utilizando el método sugerido por Fedorov (1978), basado en el análisis isentrópico.

Los muestreos efectuados en la zona al norte de PLV frente a las bahías Tongoy y Guanaquero los días siguientes, indican que la variación de las masas de agua descritas se observa también más al norte en la zona costera.

Una discusión de la advección de la masa de agua observada se basa en la evolución de las curvas T-S. En ellas se observa que la mayor variabilidad interdiaria se concentra en los $200 \mathrm{~m}$ superficiales, y que las estaciones entre 11 y $22 \mathrm{~km}$ desde la costa presentan cambios interdiarios similares a los observados en horas a 2 millas de la costa muy cerca de bahía Herradura (Moraga, 1989). Existe una variación explicable por la intrusión de masas de agua, 

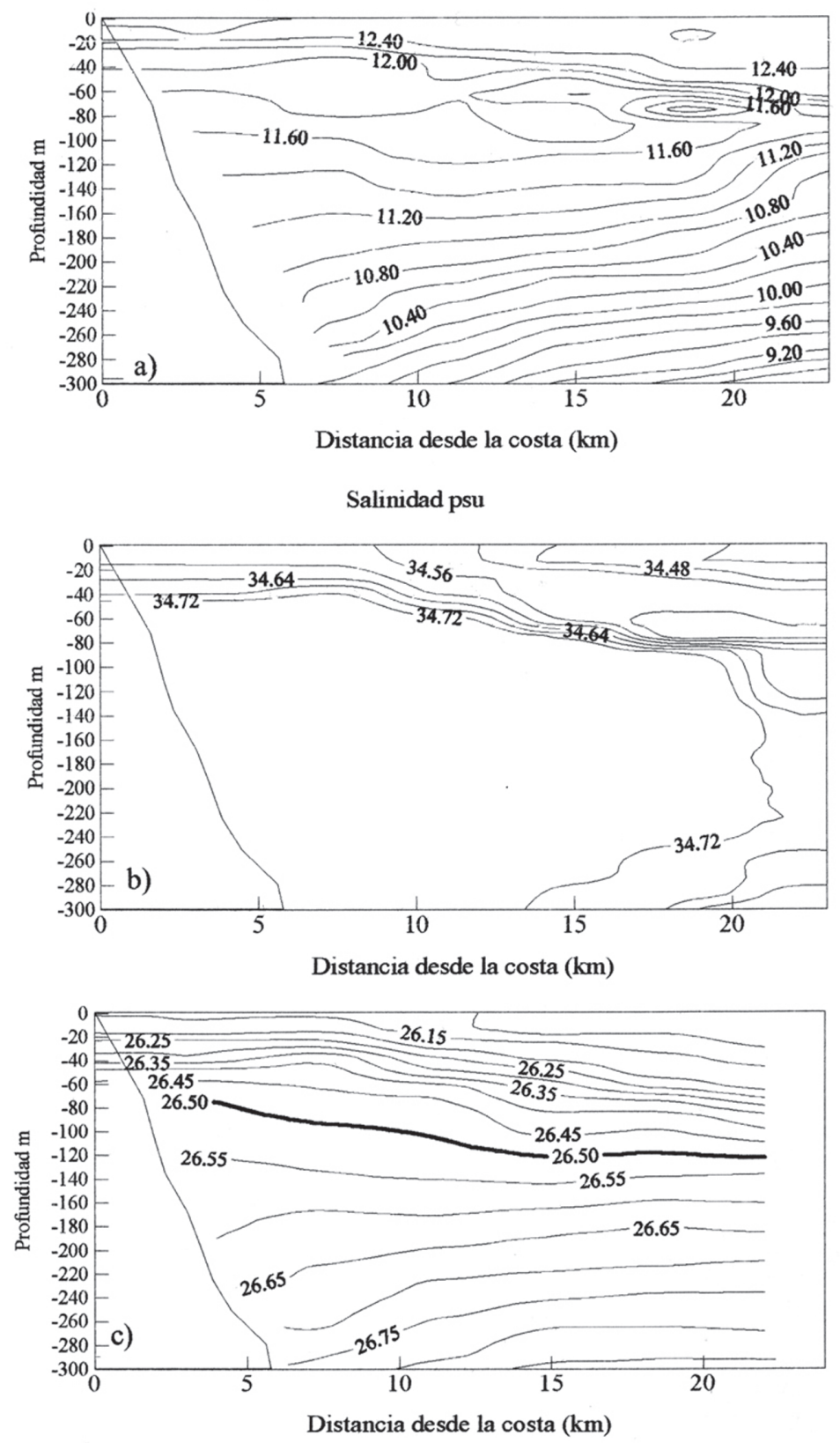

Figura 7. Distribución de variables para el día 14 en el corte $B$, frente a Punta Guanaquero: a) Isotermas, b) Isohalinas y c) Isopicnas.

Figure 7. Variable distribution on August 14, section B, in front of Point Guanaquero: a) Isotherms, b) Isohalines and c) Isopicnals. como se observa en los cortes transversales de los campos térmicos y salinos respecto a densidad potencial, cambios que no son tan evidentes como los descritos por Moraga et al. (1994) $30 \mathrm{~km}$ más al norte durante El Niño 92. Pero además, parte de la variabilidad observada puede interpretarse como el paso de ondas internas de gravedad, las cuales modifican la profundidad de ubicación de las isotermas e isohalinas.

La pendiente de las isolíneas de densidad potencial, muestra la presencia de flujos bien definidos en direcciones contrarias, separados por la isopicna de 26,5 que se mantiene aproximadamente a los $140 \mathrm{~m}$. La determinación de la capa de Ekman en el sentido clásico se puede estimar considerando el arrastre del viento y las condiciones de estratificación observadas, en este caso entre 16 y 19 m de profundidad; de igual forma, la estimación del radio de deformación de Rossby discutido por Fonseca (1985) y Johnson et al. (1980) y recientemente por Moraga et al. (1994), lleva a un valor similar de $16 \mathrm{~km}$.

Una discusión sobre el nivel de referencia para el cálculo de las velocidades geostróficas en zonas costeras puede hacerse en función de los registros directos de corrientes, método aplicado por Huyer y Kosro (1987). Pero un análisis de la variación temporal del campo de masa permite hacer algunas aproximaciones dinámicas. Si se elige como nivel de referencia para el primer día la profundidad de la isopicna de 26,5, se distingue un flujo hacia el norte en la capa superficial, y otro hacia el sur bajo los $140 \mathrm{~m}$ entre las estaciones a 8 y $15 \mathrm{~km}$. Estas dos estaciones es- 

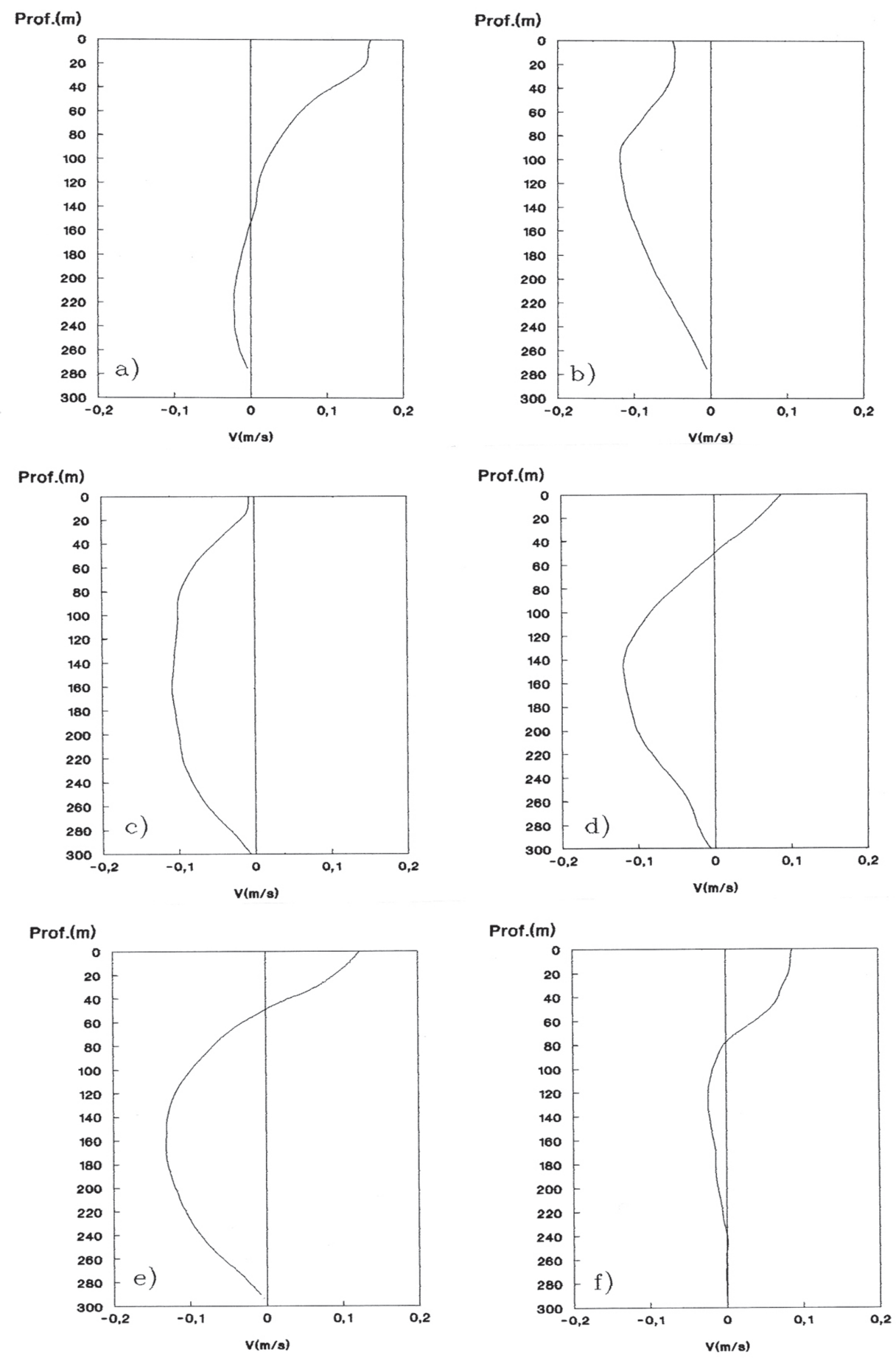

Figura 8. Corrientes geostróficas calculadas entre estaciones 4-8 mn, centradas en 6 mn (7,4 -14,8 km), 8-12 mn centradas en $10 \mathrm{mn}(14,8-22,2 \mathrm{~km})$, usando como referencia $300 \mathrm{db}$, para los siguientes días de agosto de 1990 y distancia de la costa. a) 10-6 mn; b) 10-10 mn, c) 11-6 mn; d) 11-10 mn; e) 12-6 mn; f) 12-10 mn.

Figure 8. Geostrophic currents estimated between stations at 4-8 $\mathrm{nm}$ (centered at $6 \mathrm{~nm}$ ), 8-12 $\mathrm{nm}$ (centered at 10 $\mathrm{nm}$ ), using $300 \mathrm{db}$ as level of reference, for August 1990 days and offshore distances: a) 10-6 nm; b) 10-10 nm; c) 11-6 nm; d) 11-10 nm; e) 12-6 nm; f) 12-10 nm. 
tán ubicadas lejos del borde costero, y la profundidad al fondo es mucho mayor que el límite de muestreo. La utilización de la misma aproximación para los otros días, confirmaría la alta variabilidad de los flujos norte-sur, sobre todo en los $140 \mathrm{~m} \mathrm{su}-$ perficiales. Los valores de velocidad parecen razonables y también la presencia de flujos norte-sur que varían en profundidad, esto se ve confirmado en el análisis de las pendientes de las isopicnas (Fig. 4).

Por otra parte, si se considera la variabilidad de los flujos geostróficos usando como referencia 300 $\mathrm{m}$ y limitando las estimaciones a las dos secciones, se refleja la influencia del forzante atmosférico (presión y magnitud del viento) en el equilibrio dinámico del campo de masa, y la influencia topográfica (generadora de vorticidad relativa). Una estimación de esta vorticidad se obtiene del análisis de la variación temporal de la isotermas e isohalinas en el análisis isentrópico, como de la variación de los flujos geostróficos (Moraga et al., 1994). La estimación de la vorticidad es aplicada por los autores en los $30^{\circ} \mathrm{S}$, unos $25 \mathrm{~km}$ más al norte, donde las condiciones oceanográficas son diferentes, pues ese sector no se ubica en la zona en que según las imágenes de satélite se localiza el foco de surgencia (Fonseca y Farías, 1987; Acuña et al., 1989).

La influencia del paso de una onda interna de gravedad de período mayor al inercial, se observa en las variaciones de la profundidad de la isopicna de 26,5. La evolución de las bajas costeras y su desplazamiento atrapada al borde costero, se ha asociado como relación causa-efecto con la propagación de ondas atrapadas a la costa en el océano con el mismo período en la costa sur occidental de Africa (Jury et al., 1990). No es posible discutir la naturaleza de esta onda con los datos analizados, siendo el forzamiento por el paso de una baja costera, sólo una hipótesis discutida con anterioridad por Jury y Brundrit (1992). Un análisis de las ondas atrapadas a la costa frente a PLV fue presentado por Schaffer et al., (1995) refiriéndose a las ondas asociadas al Niño. Estas ondas internas fueron descritas para la zona en un estudio de corto período en un lugar fijo (Moraga, 1989), y han sido observadas en un corte desde la costa hasta $24 \mathrm{mn}$ frente a los $30^{\circ} \mathrm{S}$ donde se puede reconocer el decaimiento de la amplitud de la onda con la distancia a la costa. En este estudio, la influencia de ondas internas de gravedad se observa con mayor claridad en las estaciones alejadas de la costa, pues la intrusión de masas de agua no explica toda la variabilidad observada. Las estaciones más costeras tienen una dinámica diferente de aquellas más alejadas por el efecto topográfico, lo cual produce un levantamiento de la masa de agua Ecuatorial Subsuperficial (masa de agua A), que explica su entrada a las bahías Tongoy y Guanaquero. La dominancia del arrastre del viento sobre la fricción del borde y del fondo, en las estaciones más alejadas, y de Coriolis (deriva de Ekman y efecto inercial) hace que la variabilidad temporal y espacial de las masas de agua sea mayor en las estaciones más oceánicas. Además, la influencia de los vientos locales en la dinámica es probada por la variabilidad observada en las masas de agua y en la circulación geostrófica (y aplicación simplificada de la ecuación del viento térmico).

La evolución del proceso de surgencia en invierno no debería ser muy diferente al observable en otras estaciones del año, pues la periodicidad de los vientos favorables a la surgencia es de 7-8 días. Por otra parte, la variación de la presión local y su anticipación a la intensificación de los vientos favorables a la surgencia permitiría pronosticar la iniciación de la misma, lo cual tiene gran relevancia en la productividad de la zona, especialmente en las bahías Tongoy y Guanaquero, próximas al foco de surgencia. Moraga et al., (1999) plantean que la entrada de aguas a la bahía Guanaquero durante la surgencia activa responde a este esquema, es decir, entrarían aguas con un mínimo salino desde el sur-oeste a nivel superficial y a los $60 \mathrm{~m}$, aguas de características ecuatoriales subsuperficiales del noroeste.

Aunque los resultados descritos no sean probatorios de la bifurcación de las corrientes superficiales durante la surgencia activa descrita para el área al norte de la bahía de Monterey (Rosenfeld et al., 1994), en este estudio pueden encontrarse indicios de tal circulación. La falta de registros directos de corrientes y estaciones más alejadas de la costa, no permite probar tal dinámica. Sin embargo, la variabilidad de las corrientes geostróficas estimadas, las diferencias entre las estaciones costeras y oceánicas, junto con el desplazamiento hacia el noroeste de las aguas frías del foco de surgencia observada en las imágenes satelitales (Fonseca y Farías, 1987; Acuña et al., 1987), permiten suponer que se cumplen los fundamentos teóricos aplicados por los autores del estudio frente a Monterey.

\section{CONCLUSIONES}

La surgencia observada durante este período de estudio en la zona frente a Punta Lengua de Vaca co- 
rresponde a un período de relajación e intensificación del viento. La relajación corresponde a los días 10 a 12 y los siguientes a una intensificación. El ciclo de vientos de 7-8 días domina el espectro, validando el tiempo elegido de muestreo.

Los cambios en las masas de agua son causados parcialmente por la instrusión de aguas de características diferentes a las residentes de la capa superficial de la columna de agua $(150 \mathrm{~m})$ y entre 15 y 22 $\mathrm{km}$ desde la costa, y además se deben a la influencia del paso de las ondas la que produce esas variaciones transientes propias de la dinámica ondulatoria.

\section{AGRADECIMIENTOS}

Se agradece el apoyo económico de la Universidad Católica del Norte a través del Proyecto DGI 65/09; de la Universidad de Chile (Programa de Desarrollo en Ciencias del Mar a través del proyecto "Interacción océano-atmósfera, y productividad primaria a la latitud $30^{\circ} 16^{\prime} \mathrm{S}$ ") y al FONDAPHUMBOLDT. Además, un especial agradecimiento a Wildo Argandoña por su apoyo computacional, a Arnoldo Valle-Levinson por la discusión de los contenidos, y a los revisores anónimos por la notable dedicación a la hora de realizar oportunas sugerencias para mejorar el trabajo.

\section{REFERENCIAS}

Acuña, E., J. Moraga y E. Uribe. 1989. La zona de Coquimbo: Un sistema nerítico de surgencia de alta productividad. Com. Perm. Pacífico Sur (Número Especial): 145-157.

Fedorov, K.N. 1978. The termohaline finestructure of the ocean. Pergamon Press, London, 170 pp.

Fisher, J., H. Leach y J.D.Woods. 1989. A synoptic map of isopycnic potential vorticity in the seasonal thermocline. J. Phys. Oceanogr., 19: 519-531.

Fonseca, T. 1985. Física de las aguas costeras de la zona Central de Chile. Tralka, 4(2): 337-354.

Fonseca, T. y M. Farías. 1987. Estudio del proceso de surgencia en la costa chilena utilizando percepción remota. Invest. Pesq., Chile, 34: 33-46.
Huyer, A. y P.M. Kosro. 1987. Mesoscale surveys over the shelf and slope in upwelling regions near Point Arena, California. J. Geophys. Res., 92: 1655-1681.

Johnson, D.R., T. Fonseca y H. Sievers. 1980. Upwelling in the Humboldt Coastal current near Valparaíso, Chile. J. Mar. Res., 38: 1-36.

Jury, M.R., C.I. MacArthur y C.J. Reason. 1990. Observations of trapped waves in the atmosphere and ocean along the coast of Southern Africa. S. Afr. Geogr. J., 72: 33-46.

Jury, M. y G.B. Brundrit. 1992. Temporal organization of upwelling in the Southern Benguela Ecosystem by resonant coastal trapped waves in the ocean and atmosphere. S. Afr. J. Mar. Sci., 12: 219-224.

Mellor, G.L. 1996. Introduction to Physical Oceanography. Springer Verlag, Berlin, 260 pp.

Moraga, J. 1989. Observación de ondas internas frente a Coquimbo, Chile. Cienc. Tecnol. Mar., 13: 3-17.

Moraga, J. 1996. Capa límite marina en la zona costera frente a Chile en $\operatorname{los} 29,5^{\circ} \mathrm{S}$. Estud. Oceanol., 15: $17-28$.

Moraga, J. y J. Olivares. 1993. Condiciones oceanográficas del área próxima a la costa frente a Coquimbo, Chile. Publ. Ocas. Facultad Ciencias del Mar. U.C. del Norte, Coquimbo, 2: 125-140.

Moraga, J., A. Valle-Levinson y J.L. Blanco. 1994. Hidrografía y dinámica de la capa superior del océano en la zona costera del Pacífico Suroriental $\left(30^{\circ} \mathrm{S}\right)$. Invest. Pesq., Chile, 38: 55-73.

Moraga, J., A. Valle-Levinson, J. Olivares, W. Argandoña y J.L. Blanco. 1999. Circulación dipolar en Bahía Guanaquero, IV Región, Chile. XIX Congreso de Ciencias del Mar, Antofagasta, Chile. p. 146.

Neshyba, S. y R. Méndez. 1976. Análisis de temperaturas superficiales del mar como indicadores de movimientos de aguas superficiales en el Pacífico Sur-Este. Rev. Com. Perm. Pacífico Sur, 5: 129137.

Pizarro, O., S. Hormazábal, A. González y E. Yáñez. 1994. Variabilidad del viento, nivel del mar y temperatura en la costa norte de Chile. Invest. Mar., Valparaíso, 22: 85-101. 
Rosenfeld, L.K., F.B. Schwing, N. Garfield y D.E. Tracy. 1994. Bifurcated flow from an upwelling center: a cold water source for Monterey Bay. Cont. Shelf. Res., 14: 931-964.

Shaffer, G., S. Salinas, O. Pizarro, A. Vega y S. Hormazábal. 1995. Currents in the deep ocean off Chile (30ㅇ). Deep-Sea Res., 42: 425-436.
UNESCO. 1981. Tenth report of the joint panel on oceanographic tables and standards. Tech. Pap. Mar. Sci., 36, 24 pp.

Valdebenito, E. 1997. Condiciones oceanográficas frente a Punta Lengua de Vaca $\left(30^{\circ} 16^{\prime}\right.$ S $)$ y su relación con la abundancia del zooplancton (invierno 1990). Tesis para optar al Título de Biólogo Marino. Universidad Católica del Norte. 
